\title{
ПОЛИНОМИАЛЬНЫЕ МЕТОДЫ ОЦЕНИВАНИЯ ПАРАМЕТРОВ СИГНАЛА НА ФОНЕ НЕГАУССОВСКИХ КОРРЕЛИРОВАННЫХ ПОМЕХ
}

\author{
В.В. Палагин, А.В. Ивченко, Е.А. Палагина, Д.А. Ведерников
}

Черкасский государственный технологический университет, бул. Шевченко, 460, Черкассы, 18005, Украина; e-mail: palahin@ukr.net

\begin{abstract}
При классической постановке задачи статистической оценки параметров сигналов широкое распространение получили математические модели в виде случайных процессов, имеющих гауссовский закон распределения. В реальных технических системах исследуемые случайные процессы целесообразнее рассматривать при использовании негауссовских моделей, которые в большей степени отражают реальную помеховую ситуацию. Исследования последних лет свидетельствуют о том, что при решении задач обработки негауссовских процессов перспективным является другой подход, в котором для описания статистических свойств случайных величин используются моменты и кумулянты, что позволяет с приемлемым приближением характеризовать статистические свойства негауссовых процессов. Такой подход позволяет повысить точность обработки негауссовских сигналов по сравнению с традиционным подходом при заданных ограничениях на их сложность, учесть корреляционные связи негауссовых случайных величин. В работе рассмотрены задачи моделирования процессов оценивания параметра постоянного сигнала на фоне негауссовских асимметричных коррелированных помех на основе моментно-кумулянтного описания случайных величин с использованием адаптированного метода максимизации полинома (метода Кунченко) для построения эффективных алгоритмов и компьютерных средств функционирования систем обработки сигналов. Показано, что полиномиальная обработка случайных величин, учет параметров негауссовского распределения в виде кумулянтов одномерного и многомерного распределений позволяет уменьшить дисперсию оценки оцениваемого параметра по сравнению с известными результатами.
\end{abstract}

Ключевые слова: моментно-кумулянтное описание, адаптированный метод максимизации полинома, коррелированные негауссовские стохастические процессы.

\section{Введение}

Оценивание параметров сигналов, принимаемых на фоне помех, является важной статистической задачей для многих технических систем. Для ее решения успешно применяются известные методы оценивания, в том числе метод максимального правдоподобия, метод моментов и др. [1-4]. Не смотря на то, что в общем случае применение этих методов не ограничивает использование произвольной плотности распределения случайных процессов, широкое распространение получили гауссовские плотности распределения. Это объясняется, в общем случае, не только процессами нормализации, но и удобством использования математического аппарата для получения конечных результатов. Такой подход не позволяет адекватно описать реальные случайные процессы, которые отличаются от гауссовских, что приводит к потери качества полученных оценок исследуемых случайных процессов.

Прохождение сигналов через неоднородные среды, многолучевое распространение, различные дестабилизирующие факторы способствуют возникновению случайных процессов, которые характеризуются негауссовскими плотностями распределений [5-7].

Применение традиционных методов к получению оценок параметров сигналов на фоне негауссовских помех вызывает большие трудности, связанные со сложностью их 
практической реализации, увеличением вычислительных ресурсов, что не позволяет создавать эффективных программно-алгоритмических систем обработки сигналов. При рассмотрении статистических связей негауссовских случайных процессов возникают проблемы с построением адекватных моделей случайных величин и оптимальных методов оценивания параметров.

Современные исследования показывают, что для обработки негауссовских случайных процессов перспективным является другой подход, который использует не плотности распределения, а моментное и кумулянтное описание (семиинварианты) случайных величин [7-10], что позволяет с приемлемым приближением представить статистические свойства исследуемых негауссовских случайных процессов. Это позволяет увеличить точность оценивания параметров сигналов на фоне негауссовских помех по сравнению с классическими методами при заданных ограничениях на их сложность [9-13], учесть статистические связи негауссовских случайных процессов $[14,15]$.

Применение моментного и кумулянтного описания случайных негауссовских процессов требует дальнейших теоретических и практических исследований, связанных с построением математических моделей исследуемых негауссовских процессов, учета статистических связей при применении многомерных моментов и кумулянтов, построения и анализа новых методов оценивания параметров сигналов при взаимодействии с негауссовскими помехами.

\section{Цель работы}

Цель работы заключается в создании и реализации моделей процессов оценивания параметра постоянного сигнала на фоне асимметричных коррелированных негауссовских помех на основе моментно-кумулянтного описания случайных величин и адаптированного метода максимизации полинома для синтеза эффективных методов и компьютерных средств функционирования систем обработки сигналов.

\section{Основная часть}

Моментно-кумулянтное описание многомерных негауссовских случайных процессов. Статистические зависимости между случайными величинами можно рассматривать через их многомерныемоменты и кумулянты, которые являются коэффициентами разложения многомерной характеристической функции в ряд. Статистически зависимая случайная величина характеризуется как одномерными, так и многомерными моментами, которые в общем случае непосредственно не выражаются один через другого.

Одномерные моменты $m_{i}$ i-го порядка случайной величины $\xi$ для статистически независимых выборочных значений определяются через одномерную плотность распределения $P_{1}(\xi)$ следующим выражением:

$$
E\left(\xi^{i}\right)=\int_{-\infty}^{\infty} \xi^{i} P_{1}(\xi) d x
$$

Выражение для совместных моментов случайных величин выражается через многомерную плотность распределения и имеет вид:

$$
E\left(\xi^{i}\left(t_{1}\right) \ldots \xi^{s}\left(t_{n}\right)\right)=\int \ldots . . \int \xi_{1}^{i}, \xi_{2}^{j} \ldots \xi_{n}^{s} P_{n}\left(\xi_{1} \ldots \xi_{n}, t_{1}, t_{2}, \ldots, t_{n}\right) d \xi_{1} \ldots d \xi_{n}
$$


Моменты и семиинварианты легко вычисляются по характеристической функции, где многомерная характеристическая функция, как и многомерная плотность распределения, является полным описанием случайной величины $[7,10]$. Многомерные моменты и кумулянты определяются как разложение в кратные степенные ряды многомерной характеристической функции $f_{\xi_{1}, \xi_{2}, \ldots \xi_{n}}\left(\vartheta_{1}, \vartheta_{2}, \ldots, \vartheta_{n}\right)$ [7]:

$$
m_{i, j, k, \ldots, n}=(-j)^{i+j+k+\ldots+n} \times\left[\frac{\partial^{i+j+k+\ldots+n} f_{\xi_{1}, \xi_{2}, \ldots \xi_{n}}\left(\vartheta_{1}, \vartheta_{2}, \ldots, \vartheta_{n}\right)}{\partial \vartheta_{1}^{i} \partial \vartheta_{2}^{j} \partial \vartheta_{n}^{n}}\right],
$$

а многомерные кумулянты записывается в виде:

$$
\chi_{i, j, k, \ldots n}=(-j)^{i+j+k+\ldots+n} \times\left[\frac{\partial^{i+j+k+\ldots+n} \ln f_{\xi_{1}, \xi_{2}, \ldots \xi_{n}}\left(\vartheta_{1}, \vartheta_{2}, \ldots, \vartheta_{n}\right)}{\partial \vartheta_{1}^{i} \partial \vartheta_{2}^{j} \partial \vartheta_{n}^{n}}\right] .
$$

При решении практических задач достаточной характеристикой учета статистической связи случайных величин является двухмерное вероятностное распределение $[7,14]$. В этом случае случайную величину с какой-либо статистической связью выборочных значений можно характеризовать не только одномерными, но и совместными кумулянтами, которые выражаются через совместные моменты:

$$
E\left(\xi^{i} \xi^{j}\right)=\int_{-\infty-\infty}^{\infty} \int^{\infty} \xi^{i} \xi^{j} P\left(\xi_{1}, \xi_{2}\right) d x
$$

Пусть имеются выборочные значения стационарного случайного процесса, где сами выборки можно рассматривать как отдельные случайные величины. Тогда в качестве одного из простых и распространенных случаев статистически зависимых выборочных значений можно взять взаимосвязь между двумя случайными величинами, что равносильно рассмотрению двумерной совместной плотности распределения двух случайных величин.

Рассмотрим случай, когда имеются две зависимые случайные величины $\xi$ и $\eta$ с плотностями распределения $p_{\xi}$ и $p_{\eta}$ соответственно. Согласно [7], начальные моменты первого порядка случайных величин $\xi$ и $\eta$ будут соответственно равны:

$$
m_{i}^{(\xi)}=E \xi^{i}=\int_{-\infty}^{+\infty} x^{i} p_{\xi}(x) d x, \quad m_{i}^{(\eta)}=E \eta^{i}=\int_{-\infty}^{+\infty} y^{i} p_{\eta}(y) d y .
$$

Так как случайные величины $\xi$ и $\eta$ зависимые, то кроме начальных моментов они имеют смешанные (совместные) моменты различной размерности.

Определение 1. Смешанным (совместным) моментом двух случайных величин размерности $(i, j)$ будем называть величину, равную математическому ожиданию произведения $i$-й степени случайной величины $\xi$ и $j$-й степени случайной величины $\eta$, т.e.

$$
m_{i, j}^{(\xi, \eta)}=E \xi^{i} \eta^{j}=\int_{-\infty}^{+\infty} x^{i} y^{j} p(x, y) d x d y
$$

где $p(x, y)$ - совместное распределение случайных величин $\xi$ и $\eta$.

Очевидно, имеет место равенство: 


$$
m_{i}^{(\xi)}=\mathrm{m}_{i, 0}^{(\xi, \eta)}, m_{j}^{(\eta)}=m_{0, j}^{(\xi, \eta)}, m_{i+j}^{(\xi)}=m_{i, j}^{(\xi, \xi)}, m_{i+j}^{(\eta)}=m_{i, j}^{(\eta, \eta)}
$$

Аналогично можно определить смешанный (совместный) момент трех и более случайных величин $\xi, \eta, \gamma$ размерностью $(i, j, k)$, который будет равен:

$$
m_{i, j, k}^{(\xi, \eta, \gamma)}=E \xi^{i} \eta^{j} \gamma^{k}=\int_{-\infty-\infty}^{+\infty} \int_{-\infty}^{+\infty} \int_{-\infty}^{+\infty} x^{i} y^{j} z^{k} p(x, y, z) d x d y d z
$$

Кроме начальных моментов $i$-го порядка и смешанных моментов размерностью $(i, j)$ будем использовать корреляционные моменты размерностью $(i, j)$.

Определение 2. Корреляционным моментом двух случайных величин $\xi$ и $\eta$ размерности $(i, j)$ будем называть величину $K_{i, j}^{(\xi, \eta)}$, которая определяется равенством:

$$
K_{i, j}^{(\xi, \eta)}=E\left(\xi^{i}-m_{i}^{(\xi)}\right)\left(\eta^{j}-m_{j}^{(\eta)}\right)=E \xi^{i} \eta^{j}-m_{i}^{(\xi)} m_{j}^{(\eta)}=m_{i, j}^{(\xi, \eta)}-m_{i}^{(\xi)} m_{j}^{(\eta)}
$$

Аналогично можно определить корреляционный момент для трех случайных величин размерности $(i, j, k)$, где может иметь место два случая, а именно:

$$
\begin{gathered}
\text { 1) } K_{i, j, k}^{(\xi, \eta, \gamma)}=E\left(\xi^{i}-m_{i}^{(\xi)}\right)\left(\eta^{j}-m_{j}^{(\eta)}\right)\left(\gamma^{k}-m_{k}^{(\gamma)}\right)= \\
m_{i, j, k}^{(\xi, \eta, \gamma)}-m_{k}^{(\gamma)} m_{i, j}^{(\xi, \eta)}-m_{j}^{(\eta)} m_{i, k}^{(\xi, \gamma)}-m_{i}^{(\xi)} m_{j, k}^{(\eta, \gamma)}+2 m_{i}^{(\xi)} m_{j}^{(\eta)} m_{k}^{(\gamma)}, \\
\text { 2) } K_{i, j, k}^{(\xi, \eta, \gamma)}=E\left(\xi^{i} \eta^{j}-m_{i, j}^{(\xi, \eta)}\right)\left(\gamma^{k}-m_{k}^{(\gamma)}\right)=m_{i, j, k}^{(\xi, \eta, \gamma)}-m_{i, j}^{(\xi, \eta)} m_{k}^{(\gamma)} .
\end{gathered}
$$

Аналогично могут быть определены корреляционные моменты для четырех, пяти и более случайных величин.

Использование совместных моментов позволяет оперировать не только с такими параметрами, как асимметрия $\left(\gamma_{3}\right)$, эксцесс $\left(\gamma_{4}\right)$ и другими кумулянтными коэффициентами, которые описывают характер негауссовского распределения случайной величины, но и степень взаимосвязи случайных величин.

В работах $[11,14]$ рассматривались вопросы использования на практике моделей корреляционных функций, их взаимосвязи и свойств, а также особенности использования моментно-кумулянтного описания статистически зависимых случайных величин. Показано $[9,10]$, что для негауссовых статистически независимых случайных величин с нулевым математическим ожиданием взаимосвязь между начальными моментами $\alpha_{i}$ и кумулянтами $\chi_{i}$ до шестого порядка имеет вид:

$$
\begin{gathered}
\alpha_{1}=0, \alpha_{2}=\chi_{2}, \alpha_{3}=\chi_{3}, \alpha_{4}=\chi_{4}+3 \chi_{2}^{2}, \alpha_{5}=\chi_{5}+10 \chi_{2} \chi_{3}, \\
\alpha_{6}=\chi_{6}+15 \chi_{2} \chi_{4}+10 \chi_{3}^{2}+15 \chi_{2}^{3} .
\end{gathered}
$$

Отметим, что для гауссовских случайных величин кумулянты третьего и выше порядка $\left(\chi_{3}, \chi_{4}, \ldots\right)$ равны нулю.

Если рассматривать негауссовскую статистически зависимуюслучайную величину с нулевым математическим ожиданием, то связь между совместными моментами $m_{i j}$ и кумулянтами $\chi_{i j}$ до шестого порядка имеет вид [7]: 


$$
\begin{gathered}
m_{11}=\chi_{11}, m_{12}=\chi_{12}, m_{13}=\chi_{13}+3 \chi_{2} \chi_{11}, m_{22}=\chi_{22}+\chi_{2}^{2}+2 \chi_{11}^{2}, \\
m_{23}=\chi_{23}+\chi_{2} \chi_{3}+6 \chi_{11} \chi_{12}+3 \chi_{2} \chi_{12}, \\
m_{33}=\chi_{33}+3 \chi_{31} \chi_{2}+\chi_{3}^{2}+9 \chi_{11} \chi_{22}+9 \chi_{12}^{2}+3 \chi_{13} \chi_{2}+9 \chi_{2}^{2} \chi_{11}+6 \chi_{11}^{3}, \ldots
\end{gathered}
$$

Если случайная величина является статистически независимой, то многомерные моменты трансформируются в произведение одномерных.

Моменты могут быть получены в результате экспериментальных исследований как определенные усреднения выборочных значений и оставаться единственными величинами, которые представляют определенные свойства случайной величины. Как известно, на основе бесконечной последовательности моментных функций можно восстановить вид характеристической функции. Для случайных величин, которые отличаются от гауссовских, именно эти характеристики могут нести все возможны сведения об их статистических связях и распределениях.

Так как не всегда удается иметь полную информацию о многомерной функции распределения, целесообразным является более детальное рассмотрение статистически зависимых случайных величин с помощью усредненных характеристик в виде моментов и кумулянтов. Причем отличие от нулевого значения совместных кумулянтов свидетельствует о существовании статистической зависимости между соответствующими случайными величинами.

Между совместными моментами и кумулянтами существуют связи, подобные связям для одномерных случайных величин. Известно, что совместный кумулянт второго порядка (ковариация) $\chi_{11}$ описывает статистическую связь первого порядка или коррелированность случайных величин:

$$
\chi_{11}=m_{11}-m_{\xi 1} m_{\xi 2}
$$

где $m_{\xi 1}$ и $m_{\xi 2}$ - математическое ожидание случайной величины $\xi_{1}$ и $\xi_{2}$ соответственно.

Статистическая связь между выборочными значениями, при которых $\chi_{11} \neq 0$, характеризует корреляцию этих выборочных значений. Известно, что равенство $\chi_{11}$ нулю еще не свидетельствует о статистической независимости, так как кумулянты высших порядков могут отличаться от нуля и устанавливать более сложную зависимость между случайными величинами, чем их зависимость при взаимной корреляции [10]. Согласно этих рассуждений, кумулянт третьего порядка описывает статистическую связь второго порядка, кумулянт $s$-го порядка описывает статистическую связь $(s-1)$-го порядка. Причем эта связь может существовать независимо от наличия статистических связей низших порядков. Эту закономерность можно увидеть из математических выражений многомерных кумулянтов.

Адаптированный метод максимизации полинома и его использование для определения оценок параметров сигналов, принимаемых на фоне коррелированных негауссовских помех. Пусть в течении времени $[0, T]$ наблюдается сигнал $\xi(t)$, который представляет аддитивную смесь полезного сигнала $S(\vartheta)$ и асимметричную негауссовскую коррелированную помеху $\eta(t)$, т.е.

$$
\xi(t)=S(\vartheta)+\eta(t)
$$


Будем считать, что за время наблюдения сигнал $S(\vartheta)$ является постоянным, но его значение зависит от неизвестного параметра $\vartheta$. Предположим, что асимметричная негауссовская коррелированная помеха имеет двухмоментное распределение, является стационарной и характеризуется кумулянтами и кумулянтными функциями отличными от нуля: $\chi_{2}, \chi_{3}, \chi_{2}(0, \tau), \chi_{3}(0, \tau, \tau), \chi_{3}(0,0, \tau)$.

Пусть из наблюдаемого сигнала $\xi(t)$ осуществляется выборка $\vec{x}=\left\{x_{1}, x_{2}, \ldots x_{n}\right\}$ объемом $n$, которая является статистически зависимой, одинаково распределенной и равна:

$$
x_{v}=S_{\vartheta}+\eta_{v},
$$

где для краткости обозначим $S_{\vartheta}=S(\vartheta)$.

Необходимо по результатам обработки выборочных значений $\vec{x}=\left\{x_{1}, x_{2}, \ldots x_{n}\right\}$ найти оценку значения параметра постоянного сигнала $\vartheta$ при условии, что все остальные параметры известны.

Для решения данной задачи оценивания параметра постоянного сигнала на фоне асимметричных негауссовских коррелированных помех предлагается использование метода максимизации полинома (ММП) (метод Кунченко) $[9,10]$ и его адаптированный вариант, который успешно используется для решения многих статистических задач $[11,14]$.

Адаптированный ММП [11, 14, 15] позволяет учесть статистическую связь выборочных значений в виде совместных кумулянтов и моментов случайных величин и синтезировать полиномиальные алгоритмы оценивания параметров сигналов с меньшей дисперсией оценки по сравнению с известными результатами.

Показано, что в соответствии с адаптированным методом максимизации полинома (АММП) исследуемые выборочные значения могут бать представлены в виде стохастического полинома степени $s[11,14]$. В этом случае оценка параметра $\vartheta$ при моментно-кумулянтном описании случайного процесса будет находится из решения уравнения:

$$
\left.\sum_{i=1}^{s} h_{i(v, k)}[\vartheta] \sum_{v=1}^{n}\left(\xi_{(v, k)}^{i}-\alpha_{i}[\vartheta]\right)\right|_{\vartheta=\hat{\vartheta}}=0, v, k=\overline{1, n}
$$

где $\xi_{(v, k)}^{i}$ - статистически зависимые и одинаково распределенные выборочные значения исследуемого случайного процесса $\xi(t)$ в моменты времени $v$ и $k, \alpha_{i}[\vartheta]-$ моменты $i$-го порядка одномоментного распределения случайного процесса; $h_{i(v, k)}[\vartheta]-$ неизвестные коэффициенты, зависящие не только от неизвестного параметра $\vartheta$, который оценивается, но и от функции корреляции $r_{\xi}(\tau)$, определяемой как $\tau=|v-k|$. Нахождение оценки параметра $\vartheta$ находится из решения системы алгебраических уравнений:

$$
\sum_{i=1}^{s} h_{i(v, k)}[\vartheta] K_{i, j}(\tau, \vartheta)=\frac{d}{d \vartheta} \alpha_{i}(\vartheta), i=\overline{1, s}, v, k=\overline{1, n}
$$

где $K_{i, j}(\tau, \vartheta)$ - коэффициенты, которые определены из соотношения:

$$
\left.\left.K_{i, j}(\tau, \vartheta)=E\left\{\xi_{v}^{i}-\alpha_{i}\right\rfloor \xi_{k}^{j}-\alpha_{j}\right]\right\}=E\left[\xi_{v}^{i} \xi_{k}^{j}\right]-\alpha_{i} \alpha_{j}
$$


где $\xi_{v}^{i}, \ldots, \xi_{k}^{i}-$ значения стационарного процесса в $v$-й и $k$-й моменты дискретного времени $t(t=1,2, \ldots, n)$.

Коэффициенты $K_{i, j}(\tau, \vartheta)$ зависят от совместных моментов $E\left[\xi_{v}^{i} \xi_{k}^{j}\right]$, таким образом, имеется возможность не только описать степень негауссовости случайных величин в виде одномерных моментов высших порядков, но статистические связи исследуемых процессов.

На основе использования моментно-кумулянтного описания случайных величин в виде одномерных и двухмерных функций, использования АММП, построены полиномиальные алгоритмы оценивания параметра $\vartheta$ полезного сигнала, принимаемого на фоне асимметричных негауссовских коррелированных помех.

Анализ полиномиальных алгоритмов при использовании адаптированного метода максимизации полинома. Рассмотрим построение и анализ полиномиальных алгоритмов нахождения оценки параметра $\vartheta$ постоянного сигнала при использовании АММП при степени полинома $s=1,2$. Для этого необходимо привести начальные одномерные моменты до 4-го порядка, а также совместные двумерные моменты, позволяющие описать статистические связи исследуемых случайных коррелированных асимметричных негауссовских величин.

Общие выражения для начальных одномерных моментов асимметричной негауссовской случайной величины имеют вид:

$$
\alpha_{1}=0, \alpha_{2}=\chi_{2}, \alpha_{3}=\chi_{2}^{1,5} \gamma_{3}, \alpha_{4}=3 \chi_{2}^{2},
$$

а моментные функции двухмоментного распределения запишутся как:

$$
\alpha_{2}(0, \tau)=\chi_{2}(0, \tau), \alpha_{3}(0, \tau, \tau)=\chi_{3}(0, \tau, \tau), \alpha_{4}(0, \tau, \tau, \tau)=3 \chi_{2} \cdot \chi_{2}(0, \tau) .
$$

При использовании АММП необходимо получить начальные моменты $m_{i}$ случайной величины $\xi$. Для аддитивной смеси сигнала и помехи моменты одномоментного распределения будут соответственно равные:

$$
\begin{gathered}
m_{1}=S_{\vartheta}, m_{2}=\chi_{2}+S_{\vartheta}^{2}, m_{3}=\chi_{2}^{1,5} \gamma_{3}+3 \chi_{2} S_{\vartheta}+S_{\vartheta}^{3}, \\
m_{4}=3 \chi_{2}^{2}+4 \chi_{2}^{1,5} \gamma_{3} S_{\vartheta}+6 \chi_{2} S_{\vartheta}^{2}+S_{\vartheta}^{4},
\end{gathered}
$$

а моменты двухмоментного распределения запишутся как:

$$
\begin{aligned}
m_{1}(0, \tau)= & S_{\vartheta}, m_{2}(0, \tau)=\chi_{2}(0, \tau)+S_{\vartheta}^{2}, m_{3}(0, \tau, \tau)=\chi_{3}(0, \tau, \tau)+3 \chi_{2}(0, \tau) S_{\vartheta}+S_{\vartheta}^{3}, \\
& m_{4}(0, \tau, \tau, \tau)=3 \chi_{2} \chi_{2}(0, \tau)+4 \chi_{3}(0, \tau, \tau) S_{\vartheta}+6 \chi_{2}(0, \tau) S_{\vartheta}^{2}+S_{\vartheta}^{4} .
\end{aligned}
$$

Представление центрированных коррелянтов $K_{i, j}(\tau)$ двухмоментного стационарного случайного процесса $\xi(t)$ через кумулянты и кумулянтные функции имеет вид:

$$
K_{1,1}(0, \tau)=m_{2}(0, \tau)-m_{1} m_{2}=\chi_{2}(0, \tau)=\chi_{2} r_{\xi}(\tau)
$$




$$
\begin{aligned}
& \quad K_{1,2}(0, \tau, \tau)=m_{3}(0, \tau, \tau)-m_{1} m_{2}=\chi_{3}(0, \tau, \tau)+3 \chi_{2}(0, \tau) S_{\vartheta}-S_{\vartheta} \chi_{2}=\chi_{2}^{3 / 2} \gamma_{3} r_{\xi}(0, \tau, \tau)+ \\
& \quad+\chi_{2} S_{\vartheta}\left(3 r_{\xi}(\tau)-1\right), \\
& K_{2,2}(0,0, \tau, \tau)=m_{4}(0,0, \tau, \tau)-m_{2} m_{2}=2 \chi_{2}^{2} r_{\xi}^{2}(\tau)+4 \chi_{2}^{3 / 2} \gamma_{3} r_{\xi}(0, \tau, \tau) S_{\vartheta}+\chi_{2} S_{\vartheta}^{2}\left(6 r_{\xi}(\tau)-2\right) .
\end{aligned}
$$

Для статистически независимых выборочных значений центрированные

$$
\begin{array}{lll}
\text { коррелянты } \quad \text { имеют } & \text { вид: } \quad K_{1,1}=\chi_{2}, & K_{1,2}==_{2}=\chi_{2}^{3 / 2} \gamma_{3}+2 \chi_{2} S_{\vartheta}, \\
K_{2,2}=2 \chi_{2}^{2}+4 \chi_{2}^{3 / 2} \gamma_{3} S_{\vartheta}+4 \chi_{2} S_{\vartheta}^{2} . & &
\end{array}
$$

Предположим, что корреляционная функция негауссовской помехи известна и равна $\chi_{2}(0, \tau)=r_{\xi}(\tau)$. Данный подход будет применен для корреляционной функций второго порядка, которые могут быть использованы в различных приложениях, а сами корреляционные функции могут принимать различный вид:

$$
r_{\xi}(\tau)=\sigma^{2} e^{-A|\tau|}, r_{\xi}(\tau)=\sigma^{2} e^{-A|\tau|} \cos \beta \tau, r_{\xi}(\tau)=\sigma^{2} e^{-A|\tau|}\left(\cos \beta \tau+\frac{A}{\beta} \sin \beta|\tau|\right)
$$

где $\tau=\left|t_{v}-t_{k}\right|$ - временное расстояние между выборочными значениями, которое при учете статистических связей меньше интервала корреляции $\tau=\left|t_{v}-t_{k}\right| \leq \tau_{\kappa o p}, v, k=\overline{1, n}$; $\tau_{\text {кор }}$ - время корреляции; $\sigma^{2}=r_{\xi}(0)$ - дисперсия случайного процесса; $\frac{1}{A}>0$ постоянная времени, характеризующая статистическую связь выборочных значений. Экспериментально показано, что при $|\tau| \approx 3 \frac{1}{A}$ и более можно считать, что корреляционные связи слабые, или $r_{\xi}(\tau)=0$.

Для нахождения оценки параметра $\vartheta$ будем использовать АММП (1), коэффициенты которого находятся из системы уравнений (2). Для решения данной системы воспользуемся формулами Крамера и Шура:

$$
h_{i(v, k)}[\vartheta]=\frac{\Delta_{i s}(\vartheta)}{\Delta_{s}(\vartheta)}, i=\overline{1, s}
$$

где $\Delta_{s}(\vartheta)=\operatorname{det}\left\|K_{i, j}(\tau, \vartheta)\right\|-$ определитель матрицы размерностью $s$, элементами которой являются центральные коррелянты асимметричного коррелированного случайного процесса; $\Delta_{i s}(\vartheta)$ - определитель, получаемый из $\Delta_{s}(\vartheta)$ заменой $i$-го столбца столбцом, состоящим из свободных членов системы уравнений (2).

При степени полинома $s=1$ оценка параметра $\vartheta$ находится из уравнения (1) и примет вид:

$$
\left.\sum_{v=1}^{n} h_{1(v, k)}[\vartheta]\left(\xi_{(v, k)}-S_{\vartheta}\right)\right|_{S_{\vartheta}=\hat{S}_{\vartheta}}=0, k=\overline{1, n}
$$

Показано, что в данном случае решением уравнения (2) будет следующее равенство: 


$$
h_{1(v, k)}[\vartheta]=\frac{A_{v}(\vartheta)}{\operatorname{det}\left|K_{1,1}(\tau, \vartheta)\right|}, v, k=\overline{1, n}
$$

где $A_{v}(\vartheta)-$ определитель, получаемый из определителя $\operatorname{det}\left|K_{1,1}(\tau, \vartheta)\right|$ заменой $v$-го столбца столбцом, состоящим из свободных членов системы уравнений (2).

Выражение $K_{1,1}(\tau, \vartheta)$ представляет собой корреляционную матрицу, которая описывает статистические зависимости выборочных значений в различные моменты времени $(v, k)$ и для экспоненциальной зависимости примет вид:

$$
K_{1,1}(\tau, \vartheta)=\left|\begin{array}{cccc}
K_{1,1}^{(1,1)} & K_{1,1}^{(1,2)} & \ldots & K_{1,1}^{(1, n)} \\
K_{1,1}^{(2,1)} & K_{1,1}^{(2,2)} & \ldots & K_{1,1}^{(2, n)} \\
\cdot & \cdot & . & \cdot \\
K_{1,1}^{(n, 1)} & K_{1,1}^{(n, 2)} & \ldots & K_{1,1}^{(n, n)}
\end{array}\right|=\sigma^{2}\left|\begin{array}{cccc}
1 & e^{-A} & \ldots & e^{-A(n-1)} \\
e^{-A} & 1 & \ldots & e^{-A(n-2)} \\
\cdot & \cdot & . & \cdot \\
e^{-A(n-1)} & e^{-A(n-2)} & \ldots & 1
\end{array}\right|
$$

Подставляя выражение для оптимального коэффициента $h_{1(v, k)}[\vartheta]$ в уравнение максимизации полинома (3) получим выражение, из которого находится оценка полезного сигнала в виде:

$$
\hat{S}_{\vartheta}=\frac{\sum_{v=1}^{n} A_{v}(\vartheta) x_{v}}{\sum_{v=1}^{n} A_{v}(\vartheta)},
$$

которая при статистически независимых выборочных значениях вырождается в хорошо известный вид $[9,10]$ :

$$
\hat{S}_{\vartheta}=\frac{1}{n} \sum_{v=1}^{n} x_{v}
$$

Качество полученной оценки можно проанализировать через количество извлекаемой информации о параметре $\vartheta$ из выборочных значений, которое обратно пропорционально дисперсии оценки и в общем случае имеет вид $[9,10]$ :

$$
I_{s n}(\vartheta)=\sum_{v=1}^{n} \sum_{i=1}^{s} h_{i(v, k)}[\vartheta] \frac{d}{d \vartheta} \alpha_{i}(\vartheta)=\frac{1}{\sigma_{(\vartheta) s}^{2}}, k=\overline{1, n}
$$

Для рассматриваемого случая при степени полинома $s=1$ количество извлекаемой информации примет вид

$$
I_{1 n}(\vartheta)=\sum_{v=1}^{n} h_{1(v, k)}[\vartheta]=\frac{A_{v}(\vartheta)}{\operatorname{det} \mid K_{1,1}(\tau, \vartheta)}
$$

При рассмотрении первой степени полинома используются начальные моменты только первого и второго порядка, что не дает возможности описать негауссовское распределение исследуемых случайных величин. Поэтому увеличим степень полинома до $s=2$ и для получения оценки параметра $\vartheta$ воспользуемся уравнением (1), в котором неизвестные коэффициенты, согласно (2), примут вид: 


$$
h_{1(v, k)}[\vartheta]=\frac{\Delta_{12}(\vartheta)}{\Delta(\vartheta)}, h_{2(v, k)}[\vartheta]=\frac{\Delta_{22}(\vartheta)}{\Delta(\vartheta)}
$$

где $\left.\Delta(\vartheta)=\operatorname{det}\left\|\begin{array}{cc}K_{1,1}(0, \tau, \vartheta) & K_{1,2}(0, \tau, \tau, \vartheta) \\ K_{2,1}(0, \tau, \tau, \vartheta) & K_{2,2}(0,0, \tau, \tau, \vartheta)\end{array}\right\|, \Delta_{12}(\vartheta)=\operatorname{det} \| \begin{array}{ll}\frac{d}{d \vartheta} m_{1}(\vartheta) & K_{1,2}(0, \tau, \tau, \vartheta) \\ \frac{d}{d \vartheta} m_{2}(\vartheta) & K_{2,2}(0,0, \tau, \tau, \vartheta)\end{array}\right) \|$,

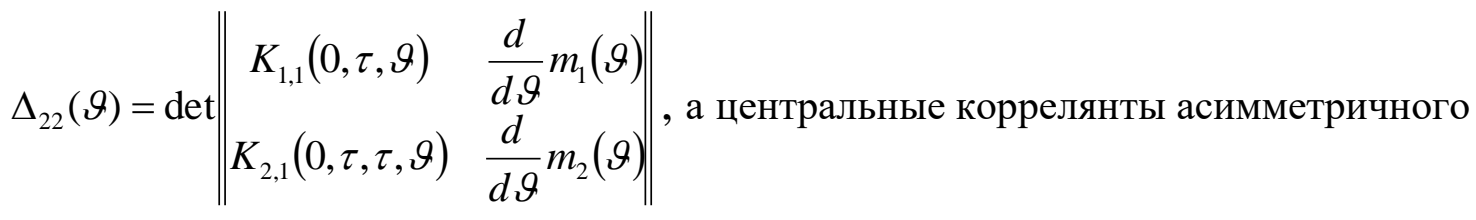
коррелированного негауссовского случайного процесса приведены выше.

Из-за громоздкости выражений значения определителей не приводится. Подставляя полученные коэффициенты в уравнение (1) получим уравнение максимизации полинома второй степени случайного процесса для нахождения оценки неизвестного параметра.

Если выборка является некоррелированной, то есть $\left(r_{\xi}(v, k)=0\right.$ если $v \neq \mathrm{k}$ и $r_{\xi}(v, k)=1$ если $\left.v=\mathrm{k}\right)$, то уравнение максимизации полинома второй степени случайного процесса примет хорошо известный вид $[9,10]$ :

$$
\gamma_{3} S_{\vartheta}^{2}-\left(2 \gamma_{3} \frac{1}{n} \sum_{v=1}^{n} x_{v}-2 \chi_{2}^{\frac{1}{2}}\right) S_{\vartheta}-\left.\left[2 \chi_{2}^{\frac{1}{2}} \frac{1}{n} \sum_{v=1}^{n} x_{v}-\gamma_{3} \frac{1}{n} \sum_{v=1}^{n} x_{v}^{2}+\chi_{2} \gamma_{3}\right]\right|_{S_{9}=\hat{S}_{9}}=0 .
$$

Легко показать, что при рассмотрении гауссовских моделей случайных процессов, когда коэффициент асимметрии $\gamma_{3}=0$, выражение (7) преобразуется к хорошо известному виду (5).

Найдем выражение дисперсии оценки постоянного сигнала для рассматриваемой модели случайного коррелированного процесса. Согласно методу максимизации полинома дисперсия оценки $\vartheta$ обратно пропорциональна количеству извлекаемой информации об оцениваемом параметре $[9,10]$ :

$$
\sigma_{(\vartheta) s}^{2}=I_{s n}^{-1}(\vartheta),
$$

где $I_{s n}(\vartheta)=\sum_{v=1}^{n} \sum_{i=1}^{s} h_{i(v, k)}[\vartheta] \frac{d}{d \vartheta} \alpha_{i}(\vartheta)=\sum_{v=1}^{n} \sum_{i=1}^{s} \sum_{j=1}^{s} h_{j(v, k)}[\vartheta] h_{i(v, k)}[\vartheta] K_{i, j}(\tau, \vartheta)$.

При построении математических моделей негауссовских коррелированных случайных процессов основным критерием оценивания качества полученных результатов (оценки неизвестного параметра), является дисперсия оценки. В этом случае отношение дисперсии оценки, найденной предложенным методом АММП при степени полинома $s=2$ к дисперсии оценки, найденной при степени полинома $s=1$, при котором не учитывается негауссовское распределение случайных величин, имеет вид:

$$
g(\vartheta)=\frac{\sigma_{(\vartheta) 2 n}^{2}}{\sigma_{(\vartheta) 1 n}^{2}}=\frac{I_{1 n}(\vartheta)}{I_{2 n}(\vartheta)}
$$

На основе полученных моделей и методов оценивания постоянного параметра сигнала, принимаемого на фоне коррелированых негауссовских помех, проведено 
имитационное моделирование, показывающее эффективность полученных результатов. На рисунке 1 приведены временные зависимости для некоррелированного (а) и коррелированного (б) асимметрично-эксцесного негауссовского процесса, полученные при использовании полигауссовской генерации выборочных значений. Видно, что при наличии корреляционных связей между выборочными значениями хаотичность полученной коррелированной случайной величины уменьшились, что объясняется наличием экспоненциальной взаимосвязи между выборочными значениями. Кроме этого приведены корреляционные поля (в, г), демонстрирующие взаимную зависимость между выборочными значениями.

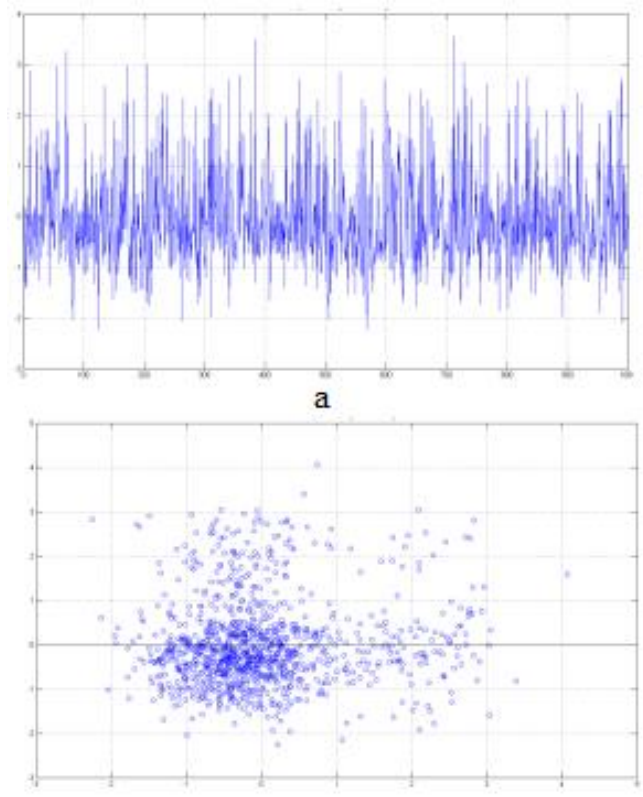

B

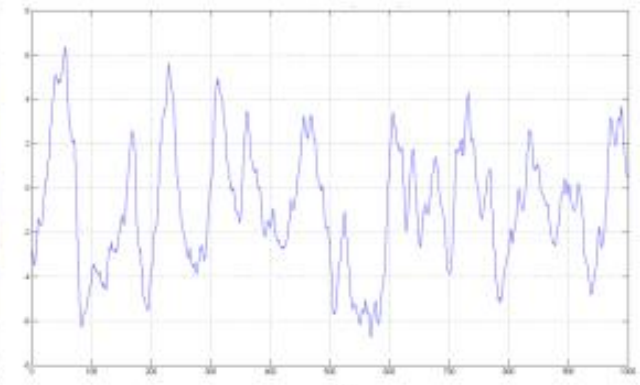

6

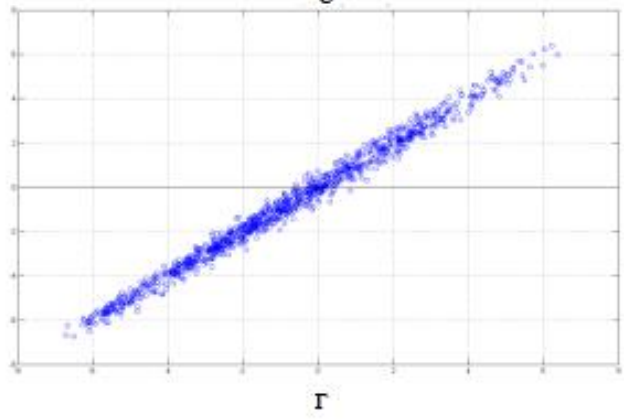

Рис. 1. Временные зависимости некоррелированного (а) и коррелированного (б) негауссовского процесса; иллюстрация корреляционных полей для некоррелированных (в) и коррелированных (г) негауссовских выборочных значений при $\mathrm{n}=1000, \mathrm{~A}=0.1$, $\gamma_{3}=0.9, \gamma_{4}=0.8$.

На рисунке 2 приведены результаты анализа полученных оценок при использовании нового метода АММП при различных степенях полинома. Проводилось оценивание зашумленого постоянного сигнала с единичной амплитудой на фоне негуссовских асимметричных коррелированных помех (а). Синтезированное правило нахождения оценки постоянного параметра (4) при степени полинома $s=1$ не учитывает негауссовский характер исследуемого случайного процесса, т.к. для его описания использовались только первые два момента, характеризующие среднее значение и дисперсию случайной величины. Для (4) найдена характеристика $I_{1}$ (6), определяющая эффективность оценивания в виде количества извлекаемой информации об оцениваемом параметре, зависимость которой от параметра корреляции А приведено на рисунке 2(б). Видно, что с ростом параметра А, что соответствует уменьшению корреляционных связей, наблюдается увеличение $I_{1}$, что свидетельствует об уменьшении дисперсии оценки. С ростом степени полинома $s=2$ (1) для нахождения оценки неизвестного параметра учитываются не только начальные моменты в виде коэффициента асимметрии $\gamma_{3}$, который характеризует степень негауссовости исследуемого случайного процесса, но совместные моменты, позволяющие описать корреляционные свойства. На рисунке 2(в, г) приведены сравнительные результаты отношения $I_{1} / I_{2}$ от значения коэффициента асимметрии $\gamma_{3}$ 
при различных значениях коэффициента корреляции $\mathrm{A}=5,0.1$. Из графиков видно, что учет параметра $\gamma_{3}$ позволяет увеличить значение количества извлекаемой информации $I_{2}$ по сравнению с $I_{1}$, что эквивалентно уменьшению дисперсии оценки неизвестного параметра. При слабых корреляционных связях $(\mathrm{A}=5)$ результат эффективности оценивания будет совпадать с хорошо изученными свойствами, представленными в [9, 10]. В тоже время необходимо отметить, что наличие сильных корреляционных связей (уменьшение коэффициента А с 5 до 0.1 для экспоненциальной корреляционной функции $\left.r_{\xi}(\tau)=\sigma^{2} e^{-A|\tau|}\right)$ между выборочными значениями уменьшает эффективность оценивания, но дисперсия оценки при нелинейной обработке $(s=2)$ все равно будет меньше по сравнению с хорошо известными результатами в предположении гауссовских моделей исследуемых случайных процессов $(s=1)$. При граничных значениях коэффициента асимметрии $\gamma_{3}=1$ уменьшение дисперсии оценки при $\mathrm{A}=5$ составляет до 2 раз, а при увеличении корреляционных связей при $\mathrm{A}=0.1$ до 1.4 раз.

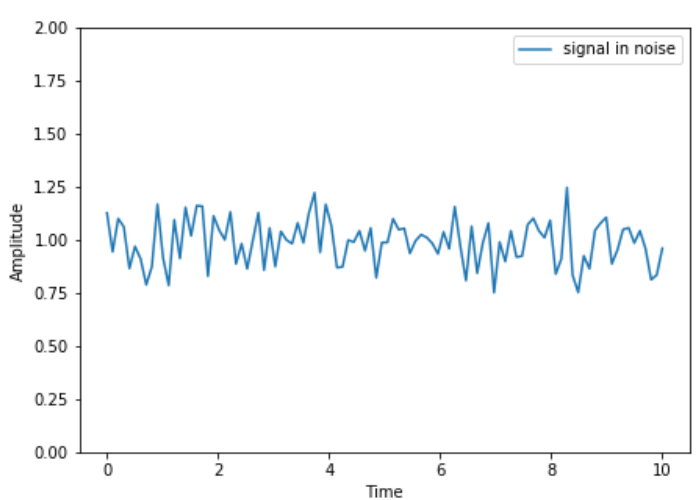

a

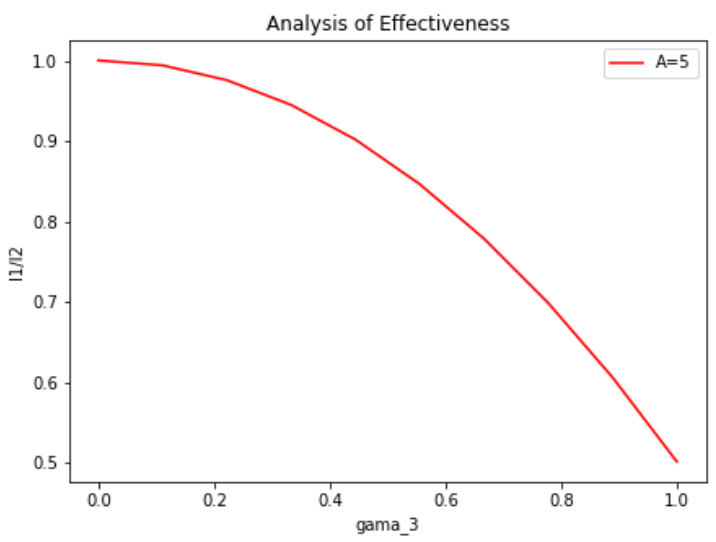

B

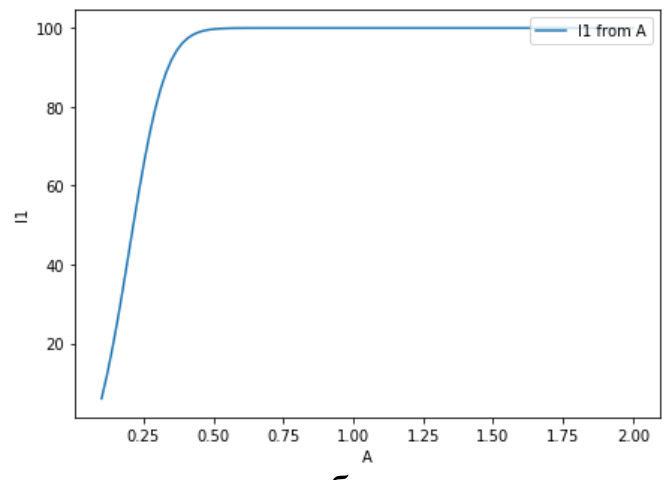

6

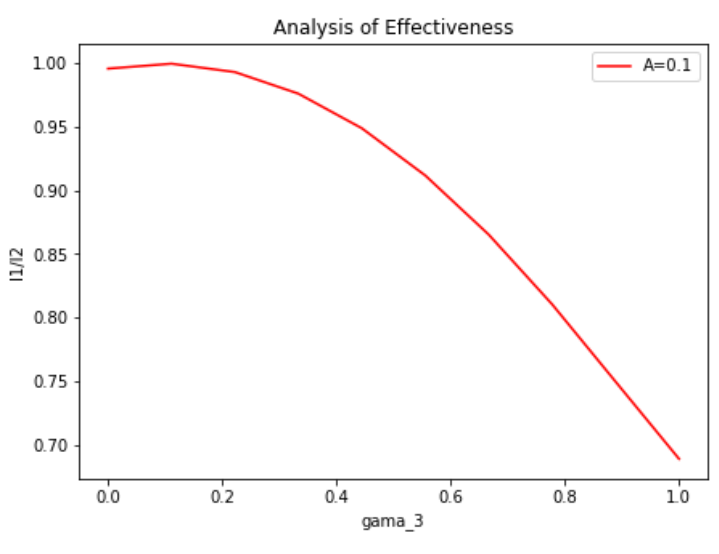

$\Gamma$

Рис. 2. Временные зависимости зашумленного постоянного сигнала с единичной амплитудой (а); зависимость количества извлекаемой информации об оцениваемом параметре сигнала от коэффициента корреляции А; сравнительные результаты отношения $I_{1} / I_{2}$ от значения коэффициента асимметрии $\gamma_{3}$ при различных значениях коэффициента корреляции $(\mathrm{A}=5,0.1)$ корреляционной функции $r_{\xi}(\tau)=\sigma^{2} e^{-A|\tau|}$.

\section{Выводы}

Построение методов математического и компьютерного моделирования процессов оценивания неизвестных параметров сигналов, принимаемых на фоне негауссовских помех при использовании моментно-кумулянтного подхода к описанию случайных процессов и адаптированного метода максимизации полинома (метод Кунченко) позволило создать алгоритмические основы обработки сигналов, что 
позволяет повысить точность оценивания параметров сигналов в компьютеризированных системах приема и обработки данных при учете параметров негауссовских помех. Использование совместных моментов и кумулянтов позволило создать новые модели коррелированных негауссовских асимметричных случайных величин и учесть корреляционные связи между ними. На основе использования адаптированного метода максимизации полинома синтезированы полиномиальные алгоритмы оценивания неизвестного параметра постоянного сигнала при взаимодействии с негауссовской помехой с меньшим значением дисперсии, что улучшает качество оценки по сравнению с хорошо известными результатами в предположении широко распространенных гауссовских помех. Эффективность полученных результатов зависит не только от степени негауссовости исследуемого случайного процесса (коэффициента асимметрии), но и от параметра, характеризующего статистические связи выборочных значений.

\section{Список литературы}

1. Van Trees H.L., Bell K.L., Tiany Z. Detection Estimation and Modulation Theory, 2nd Edition, Part I, Detection, Estimation, and Filtering Theory. New York: John Wiley \& Sons, 2013.

2. Безрук В.М., Певцов Г.М. Теоретические основы проектирования систем распознавания сигналов для автоматизированного радиоконтроля. Х.: Коллегиум, 2007. - 430 с.

3. Tuzlukov V.P. Signal Processing Noise. Boca Raton: CRC Press LLC, 2002.

4. Mourad Barkat Signal Detection and Estimation. Boston: Artech House, 2005.

5. Middleton D. Non-Gaussian Statistical Communication Theory Jonn. New Jersey: Willey \& Sons, 2012.

6. Huihong Zhao, Chenghui Zhang Non-Gaussian noise quadratic estimation for linear discrete-time time-varying systems. Neurocomputing. 2016. 174(B). Pp. 921-927.

7. Малахов А.Н. Кумулянтный анализ негауссовских процессов и их преобразований. М.: Сов. радио, 1979. 376 с.

8. Nandi A.K. Blind Estimation Using Higher-Order Statistics. New York: Springer-Verlag, 1999.

9. Кунченко Ю.П. Полиномиальные оценки параметров близких к гауссовским случайных величин. Часть I. Стохастические полиномы, их свойства и применение для нахождения оценок параметров. Черкассы: ЧИТИ, 2001. 133 с.

10. Kunchenko Y.P. Polynomial Parameter Estimations of Close to Gaussian Random variables. Aachen: Shaker Verlag, 2002. 396 p.

11. Палагін В.В., Івченко О.В. Адаптація методу максимізації полінома для оцінки параметрів випадкових величин за статистично залежною вибіркою. Системи обробки інформації. 2009. Вип. 2(76). С. 118-123.

12. Palahin V., Palahina O., Filipov V., Leleko S., Ivchenko A. Modeling of Joint Signal Detection and Parameter Estimation on Background of Non-Gaussian Noise. Journal of Applied Mathematics and Computational Mechanics. 2015. 14(3). Pp. 87-94.

13. Palahin V., Juhar J. Joint Signal parameters estimation in non-Gaussian noise by the method of polynomial maximization. Journal of Electrical Engineering. 2016. Vol.67, No.3. Pp. 217-221.

14. Vokorokos L., Marchevsky S., Ivchenko A., Palahina E., Palahin V. Parameters Estimation of Correlated non-Gaussian processes by the Method of Polynomial Maximization. Submitted to IET Signal Processing. 2016. Pp. 313-319.

15. Палагін В.В., Івченко О.В., Вєдєрніков Д.А. Знаходження оцінок параметра постійного сигналу на фоні негаусових корельованих завад. Праці VII Міжнародної науковопрактичної конферениії «Обробка сигналів і негауссівських процесів». Тези доповідей, Черкаси: ЧДТУ, 2019. С.103-106. 


\title{
ПОЛІНОМІАЛЬНІ МЕТОДИ ОЦІНЮВАННЯ ПАРАМЕТРІВ СИГНАЛІВ НА
} ФОНІ НЕГАУСОВИХ КОРЕЛЬОВАНИХ ЗАВАД

\author{
В.В. Палагін, О.В. Івченко, О.А. Палагіна, Д.А. Вєдєрніков
}

Черкаський державний технологічний університет,

бул. Шевченка, 460, Черкаси, 18005, Україна; e-mail: palahin@ukr.net

\begin{abstract}
При класичній постановці задачі статистичної оцінки параметрів сигналів широкого поширення набули математичні моделі у вигляді випадкових процесів, що мають гаусовий закон розподілу. У реальних технічних системах досліджувані випадкові процеси доцільніше розглядати при використанні негаусових моделей, які в більшій мірі відображають реальну завадову ситуацію. Дослідження останніх років свідчать про те, що при вирішенні задач обробки негаусових процесів перспективним $\epsilon$ інший підхід, в якому для опису статистичних властивостей випадкових величин використовуються моменти і кумулянти, що дозволяє з прийнятним наближенням характеризувати статистичні властивості негаусових процесів. Такий підхід дозволяє підвищити точність обробки негауссовских сигналів у порівнянні 3 традиційним підходом при заданих обмеженнях на їх складність, врахувати кореляційні зв'язки негаусових випадкових величин. В роботі розглянуто задачі моделювання процесів оцінювання параметра постійного сигналу на фоні негаусових асиметричних корельованих завад на основі моментно-кумулянтного опису випадкових величин 3 використанням адаптованого методу максимізації полінома (методу Кунченко) для побудови ефективних алгоритмів і комп'ютерних засобів функціонування систем обробки сигналів. Показано, що поліноміальна обробка випадкових величин, врахування параметрів негаусового розподілу у вигляді кумулянтних одновимірних і багатовимірних розподілів дозволяє зменшити дисперсію оцінки оцінюваного параметра у порівнянні з відомими результатами.
\end{abstract}

Ключові слова: моментно-кумулянтних опис, адаптований метод максимізації полінома, корельовані негаусові стохастичні процеси.

\section{POLYNOMIAL METHODS FOR SIGNAL PARAMETERS ESTIMATION IN NON-GAUSSIAN CORRELATED NOISE}

\author{
V.V. Palahin, A.V. Ivchenko, E.A. Palahina, D.A. Viediernikov \\ Cherkasy State Technological University, \\ 460, Shevchenko blvd., Cherkasy, Ukraine, 18005; e-mail: palahin@ukr.net
}

In the classical formulation of the problem of statistical signal parameters estimation mathematical models in the form of random processes having a Gaussian distribution law were widely used. In real technical systems, the studied random processes are more appropriate to consider when using non-Gaussian models, which to a greater extent reflect the real noise situation. Recent studies have shown that when solving the problems of processing non-Gaussian processes, another approach is promising, in which moments and cumulants are used to describe the statistical properties of random variables, which allows us to characterize the statistical properties of non-Gaussian processes with an acceptable approximation. Such an approach makes it possible to increase the accuracy of processing non-Gaussian signals in comparison with the traditional approach with given restrictions on their complexity, and to take into account the correlation relationships of non-Gaussian random variables. The problems of modeling the processes of the constant signal parameter estimation in non-Gaussian asymmetric correlated noise based on the moment-cumulant description of random variables using the adapted method of polynomial maximization (Kunchenko method) for sinthesis effective algorithms and computer tools for signal processing are considered. It is shown that polynomial processing of random variables, taking into account the parameters of a non-Gaussian distribution in the form of cumulants of one-dimensional and multidimensional distributions, makes it possible to reduce the variance of the parameter estimated in comparison with the well known results.

Keywords: moment-cumulant description, the adapted method of polynomial maximization, correlated non-Gaussian stochastic processes. 